\title{
Note \\ Natural Convection Flow of a Non-Newtonian \\ Fluid Between Two Vertical Flat Plates
}

By

K. R. Rajagopal, Pittsburgh, Pennsylvania, and T. Y. Na, Dearborn, Michigan

With 2 Figures

(Received September 15, 1983; revised April 11, 1984)

\section{Summary}

The natural convection of a homogeneous incompressible fluid of grade three is investigated between two infinite parallel vertical plates. The effect of the non-Newtonian nature of fluid on the skin friction and heat transfer are studied.

\section{Introduction}

In this note we consider the natural convection of a non-Newtonian fluid, namely the Rivlin-Ericksen fluid of grade three, between two infinite parallel vertical flat plates. The stress in such a fluid is related to the motion in the following manner (cf. Truesdell and Noll [1]):

$$
\begin{aligned}
\boldsymbol{T}= & -p \boldsymbol{l}+\mu \boldsymbol{A}_{1}+\alpha_{1} \boldsymbol{A}_{2}+\alpha_{2} \boldsymbol{A}_{1}{ }^{2}+\beta_{1} \boldsymbol{A}_{3} \\
& +\beta_{2}\left[\boldsymbol{A}_{1} \boldsymbol{A}_{2}+\boldsymbol{A}_{2} \boldsymbol{A}_{1}\right]+\beta_{3}\left(\operatorname{tr} \boldsymbol{A}_{1}{ }^{2}\right) \boldsymbol{A}_{1}
\end{aligned}
$$

where $\mu$ is the coefficient of viscosity, $\alpha_{1}$ and $\alpha_{2}$ material moduli popularly referred to as normal stress moduli. The kinematical tensors $\boldsymbol{A}_{1}, \boldsymbol{A}_{2}$ and $\boldsymbol{A}_{3}$ are defined through (cf. Rivlin and Ericksen [2]):

and

$$
\boldsymbol{A}_{\mathbf{1}}=\operatorname{grad} \boldsymbol{v}+(\operatorname{grad} \boldsymbol{v})^{T},
$$

$$
\boldsymbol{A}_{n}=\frac{d}{d t} \boldsymbol{A}_{n-1}+\boldsymbol{A}_{n-1} \boldsymbol{L}+\boldsymbol{L}^{T} \boldsymbol{A}_{n-1}, \quad n=1,2,
$$

where $\frac{d}{d t}$ denotes material time differentiation, and $\boldsymbol{L}=\operatorname{grad} \boldsymbol{v}$. 
The above model contains as a subclass the classical linearly viscous Newtonian fluid (when all material moduli except $\mu$ are zero) and the class of fluids of grade two (when the $\beta$ 's are zero).

The natural convection problem between vertical flat plates for a certain class of non-Newtonian fluids has been carried out by Bruce and $\mathrm{Na}$ [3]. Other laminar natural convection problems involving heat transfer have been studied and we refer the reader to [4] for details of the same. However, in these problems a complete thermodynamic analysis of the constitutive functions have not been carried out. For the model (1), a detailed thermodynamic study has been carried out in [5]. If the model is to be compatible with thermodynamics in the sense that all motions of the fluid meet the Clausius-Duhem inequality (which is usually considered as an interpretation of the second law of thermodynamics) and the assumption that the specific Helmholtz free energy be a minimum in equilibrium, then

$$
\mu \geqq 0, \quad \alpha_{1} \geqq 0, \quad\left|\alpha_{1}+\alpha_{2}\right| \leqq \sqrt{24 \mu \beta_{3}}, \quad \beta_{1}=\beta_{2}=0, \quad \beta_{3} \geqq 0 .
$$

Thus in the case of a thermodynamically compatible fluid of third grade (1) reduces to

$$
\boldsymbol{T}=-p \boldsymbol{l}+\mu \boldsymbol{A}_{1}+\alpha_{1} \boldsymbol{A}_{2}+\alpha_{2} \boldsymbol{A}_{1}{ }^{2}+\beta_{3}\left(\operatorname{tr} \boldsymbol{A}_{1}{ }^{2}\right) \boldsymbol{A}_{1} .
$$

For the problem under consideration, a fluid represented by (5), whose material coefficients satisfy ( 4 ), is in between two vertical flat plates a distance ' $2 b$ ' apart. The walls at $x=+b$ and $x=-b$ are held at constant temperatures $\theta_{2}$ and $\theta_{1}$ respectively, where $\theta_{1}>\theta_{2}$. This difference in temperature causes the fluid near the wall at $x=-b$ to rise and the fluid near the wall at $x=b$ to fall. In the next section we shall determine the velocity profile due to this flow.

\section{Analysis}

For the problem in question, we shall seek velocity and temperature fields of the form

$$
\boldsymbol{v}=v(x) \boldsymbol{j}, \quad \theta=\theta(x)
$$

It follows from (5), (6) and the balance of linear momentum, that

$$
\begin{gathered}
\left(2 \alpha_{1}+\alpha_{2}\right) \frac{d}{d x}\left(\frac{d v}{d x}\right)^{2}=\frac{\partial p}{\partial x}, \\
\mu \frac{d^{2} v}{d x^{2}}+6 \beta_{3}\left(\frac{d v}{d x}\right)^{2} \frac{d^{2} v}{d x^{2}}-\varrho_{0}\left[1-\gamma\left(\theta-\theta_{m}\right)\right] g=\frac{\partial p}{\partial y}, \\
0=\frac{\partial p}{\partial z} .
\end{gathered}
$$


In deriving the Eqs. $(7.1,2,3)$ the usual Boussinesq law is assumed for the body force, i.e.,

$$
\varrho b=-\varrho_{0}\left[1-\gamma\left(\theta-\theta_{m}\right)\right] g \boldsymbol{j},
$$

where $g$ denotes gravity, $\gamma$ is the coefficient of thermal expansion and $\varrho_{0}$ is a constant and $\theta_{m}$ a reference temperature which we shall pick as $\theta_{m}=\frac{1}{2}\left(\theta_{1}+\theta_{2}\right)$. Defining a modified pressure through

$$
\hat{p}=p-\left(2 \alpha_{1}+\alpha_{2}\right)\left(\frac{d v}{d x}\right)^{2},
$$

$(7.1,2,3)$ can be re-written as

$$
\begin{gathered}
0=\frac{\partial \hat{p}}{\partial x}, \\
\mu \frac{d^{2} v}{d x^{2}}+6 \beta_{3}\left(\frac{d v}{d x}\right)^{2} \frac{d^{2} v}{d x^{2}}-\varrho_{0}\left[1-\gamma\left(\theta-\theta_{m}\right)\right] g=\frac{\partial \hat{p}}{\partial y}, \\
0=\frac{\partial \hat{p}}{\partial z} .
\end{gathered}
$$

Equations $(9.1,2,3)$ imply that $\frac{\partial \hat{p}}{\partial y}$ is at most a constant. On appropriately extending the usual approximations, the equation of motion reduces to

$$
\mu \frac{d^{2} v}{d x^{2}}+6 \beta_{3}\left(\frac{d v}{d x}\right)^{2} \frac{d^{2} v}{d x^{2}}+\varrho_{0} \gamma\left(\theta-\theta_{m}\right) g=0
$$

We now proceed to derive the energy equation appropriate for the problem under consideration. We start with the energy equation

$$
\varrho \frac{d \varepsilon}{d t}=\boldsymbol{T} \cdot \boldsymbol{L}-\operatorname{div} \boldsymbol{q}+\varrho r
$$

where $\varepsilon$ is the specific internal energy, $\boldsymbol{L}$ is the gradient of velocity, $\boldsymbol{q}$ is the heat flux vector and $r$ the radiant heating. It follows from (1) that

$$
\boldsymbol{T} \cdot \boldsymbol{L}=\frac{\mu}{2}\left|\boldsymbol{A}_{1}\right|^{2}+\frac{\alpha_{1}}{4} \frac{d}{d t}\left|\boldsymbol{A}_{1}\right|^{2}+\frac{\left(\alpha_{1}+\alpha_{2}\right)}{2} \operatorname{tr} \boldsymbol{A}_{1}{ }^{3}+\frac{\beta_{3}}{2}\left|\boldsymbol{A}_{1}\right|^{4}
$$

For the problem under consideration in virtue of $(6.1), \boldsymbol{T} \cdot \boldsymbol{L}$ reduces to

$$
\boldsymbol{T} \cdot \boldsymbol{L}=\mu\left(\frac{d v}{d x}\right)^{2}+2 \beta_{3}\left(\frac{d v}{d x}\right)^{4}
$$


It has been shown in [5] that if the model (1) is to be compatible with thermodynamies then the specific Helmholtz free energy which characterizes the fluid has to take the form

$$
\psi=\psi\left(\theta, \boldsymbol{A}_{1}, \boldsymbol{A}_{2}, \boldsymbol{A}_{3}\right)=\bar{\psi}\left(\theta, \boldsymbol{A}_{1}\right)=\bar{\psi}(\theta, 0)+\frac{\alpha_{1}}{4 \varrho}\left|\boldsymbol{A}_{1}\right|^{2},
$$

and further the specific entropy is defined through

$$
\eta=-\bar{\psi}_{\theta}
$$

where the subscript denotes partial differentiation with respect to that variable. Since the specific internal energy is related to the specific Helmholtz free energy through

$$
\varepsilon=\psi+\theta \eta
$$

it follows from (14), (15), (16) that

$$
\varepsilon=\hat{\varphi}(\theta)+\frac{\alpha_{1}}{4 \varrho}\left|\boldsymbol{A}_{1}\right|^{2}-\theta \hat{\varphi}_{\theta},
$$

where

$$
\hat{\varphi}(\theta) \equiv \bar{\psi}(\theta, 0)
$$

Thus,

$$
\varrho \frac{d \varepsilon}{d t}=\varrho\left\{\frac{d}{d t}\left(\hat{\varphi}(\theta)-\theta \hat{\varphi}_{\theta}\right)+\frac{\alpha_{1}}{4 \varrho} \frac{d}{d t}\left|\boldsymbol{A}_{1}\right|^{2}\right\} .
$$

Next, note that (17) implies that

$$
\varepsilon_{\theta}=\frac{d}{d \theta}\left(\hat{\varphi}-\theta \hat{\varphi}_{\theta}\right)=-\theta \hat{\varphi}_{\theta \theta} \equiv c,
$$

where $c$ is called the specific heat. Thus

$$
\frac{d \varepsilon}{d t}=c \frac{d \theta}{d t}=0
$$

by virtue of the assumed form of the temperature field (6.2). Thus, the balance of energy (11), Eqs. (13) and (21) imply that

$$
\mu\left(\frac{d v}{d x}\right)^{2}+2 \beta_{3}\left(\frac{d v}{d x}\right)^{4}-\operatorname{div} \boldsymbol{Q}+\varrho r=0 .
$$

We shall assume that the heat flux vector $\boldsymbol{q}$ satisfies Fourier's law with a thermal conductivity constant $k$, i.e.,

$$
\boldsymbol{q}=-k \operatorname{grad} \theta
$$


Then, (6.2) implies that

$$
\operatorname{div} \boldsymbol{q}=-k \frac{d^{2} \theta}{d x^{2}}
$$

Thus, if one ignores the radiant heating, (22) and (23) yield

$$
\mu\left(\frac{d v}{d x}\right)^{2}+2 \beta_{3}\left(\frac{d v}{d x}\right)^{4}+k \frac{d^{2} \theta}{d x^{2}}=0
$$

In the next section we shall solve the system of Eqs. (10) and (24). The equations are coupled and highly non-linear. The appropriate boundary conditions are

$$
\begin{aligned}
& v=0, \quad \theta=\theta_{1} \quad \text { at } \quad x=-b \\
& v=0, \quad \theta=\theta_{2} \quad \text { at } \quad x=+b .
\end{aligned}
$$

\section{Solution}

Let us introduce non-dimensional parameters

$$
\bar{v}=\frac{v}{V_{0}}, \quad \bar{x}=\frac{x}{b}, \quad \bar{\theta}=\frac{\theta-\theta_{m}}{\theta_{1}-\theta_{2}}
$$

where $V_{0}$ is some reference velocity. Then, Eqs. (10) and (24) can be re-written as

$$
\frac{d^{2} \bar{v}}{d \bar{x}^{2}}+\frac{6 \beta_{3} V_{0}^{2}}{\mu b^{2}}\left(\frac{d \bar{v}}{d \bar{x}}\right)^{2} \frac{d^{2} \bar{v}}{d \bar{x}^{2}}+\frac{\varrho_{0} \gamma b^{2}}{\mu V_{0}} g\left(\theta_{1}-\theta_{2}\right) \bar{\theta}=0
$$

and

$$
\frac{d^{2} \bar{\theta}}{d x^{2}}+\frac{\mu V_{0}^{2}}{k\left(\theta_{1}-\theta_{2}\right)}\left(\frac{d \bar{v}}{d \bar{x}}\right)^{2}+2 \beta_{3} \frac{V_{0}^{4}}{b^{2} k\left(\theta_{1}-\theta_{2}\right)}\left(\frac{d \bar{v}}{d \bar{x}}\right)^{4}=0
$$

Let us select

$$
V_{0}=\frac{\varrho_{0} b^{2}\left(\theta_{1}-\theta_{2}\right) \gamma g}{\mu}
$$

then (27) and (28) can be further simplified to

$$
\begin{gathered}
\frac{d^{2} \bar{v}}{d \bar{x}^{2}}+6 \delta\left(\frac{d \bar{v}}{d \bar{x}}\right)^{2} \frac{d^{2} \bar{v}}{d \bar{x}^{2}}+\bar{\theta}=0 \\
\frac{d^{2} \bar{\theta}}{d \bar{x}^{2}}+E \cdot(\operatorname{Pr})\left(\frac{d \bar{v}}{d \bar{x}}\right)^{2}+2 \delta E \cdot(\operatorname{Pr})\left(\frac{d \bar{v}}{d \bar{x}}\right)^{4}=0,
\end{gathered}
$$


where

$$
E \equiv \frac{V_{0}^{2}}{c\left(\theta_{1}-\theta_{2}\right)}, \quad \operatorname{Pr}=\frac{\mu c}{k},
$$

and

$$
\delta=\frac{6 \beta_{3} \nabla_{0}^{2}}{\mu b^{2}},
$$

where $c$ is the specific heat of the fluid. The appropriate boundary conditions are

$$
\begin{array}{ll}
\bar{v}=0, & \bar{\theta}=\frac{1}{2} \quad \text { at } \quad x=-b \\
\bar{v}=0, & \bar{\theta}=-\frac{1}{2} \quad \text { at } \quad x=+b .
\end{array}
$$

The Eqs. (29) - (32) have been solved numerically.

The skin friction $S$ on the plate at $x=-b$ is directly proportional to $\frac{d \bar{v}}{d \bar{x}}$,

$$
S \sim \frac{d \bar{v}}{d \bar{x}}(-1)
$$

and the heat transfer $h$ is directly proportional to $\frac{d \bar{\theta}}{d \bar{x}}$

$$
h \sim \frac{d \bar{\theta}}{d \bar{x}}(-1) .
$$

Representative values of $\frac{d \bar{v}}{d \bar{x}}(-1)$ and $\frac{d \bar{\theta}}{d \bar{x}}(-1)$ have been provided for various values of $E, \operatorname{Pr}$ and $\delta$. The analysis seems to indicate that an increase in $\delta$ holding $E$ and $\operatorname{Pr}$ fixed increase the heat transfer slightly but decreases the skin friction. On the other hand, an increase in $E$ holding $\delta$ and $P r$ fixed tends to increase the skin friction while decreasing the heat transfer. Similarly and increase in $\mathrm{Pr}$ holding $E$ and $\delta$ fixed increases the skin friction and decreases the heat transfer.

Table 1. Variation of $\frac{d \bar{v}}{d \bar{x}}(-1)$ and $\frac{d \bar{\theta}}{d \bar{x}}(-1)$

\begin{tabular}{lllll}
\hline$\gamma$ & $E$ & $\operatorname{Pr}$ & $\frac{d \bar{v}}{d \bar{x}}(-1)$ & $\frac{d \bar{\theta}}{d \bar{x}}(-1)$ \\
\hline 0.50 & 1.0 & 1.0 & 0.1628 & -0.4966 \\
1.0 & 1.0 & 1.0 & 0.1592 & -0.4966 \\
1.0 & 2.0 & 1.0 & 0.1593 & -0.4932 \\
1.0 & 4.0 & 1.0 & 0.1596 & -0.4863 \\
2 & 1.0 & 0.1 & 0.1532 & -0.4997 \\
2 & 1.0 & 1.0 & 0.1533 & -0.4967 \\
\hline
\end{tabular}




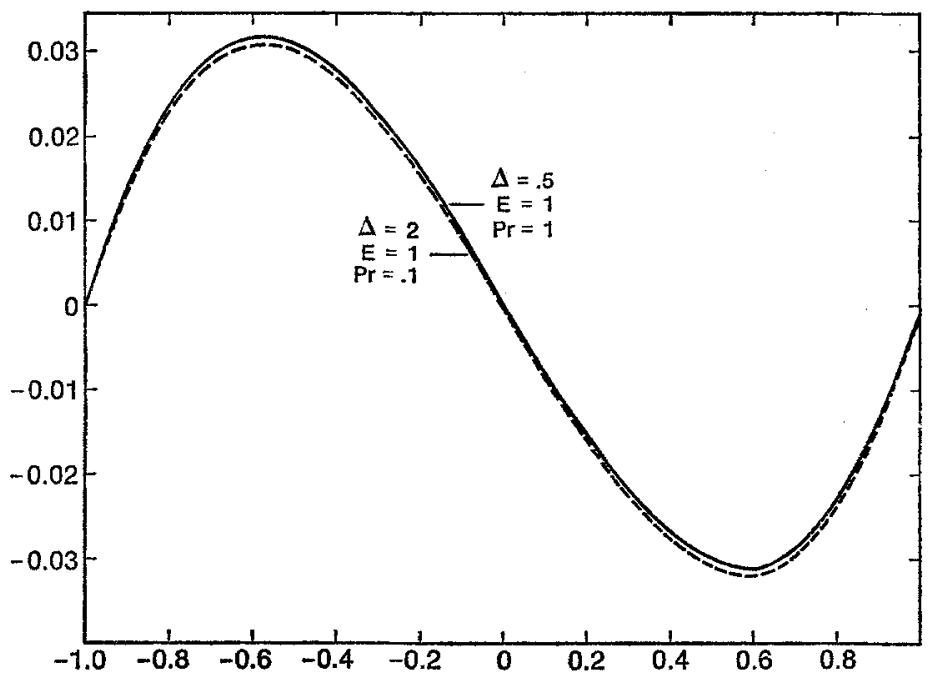

Fig. 1. Variation of velocity

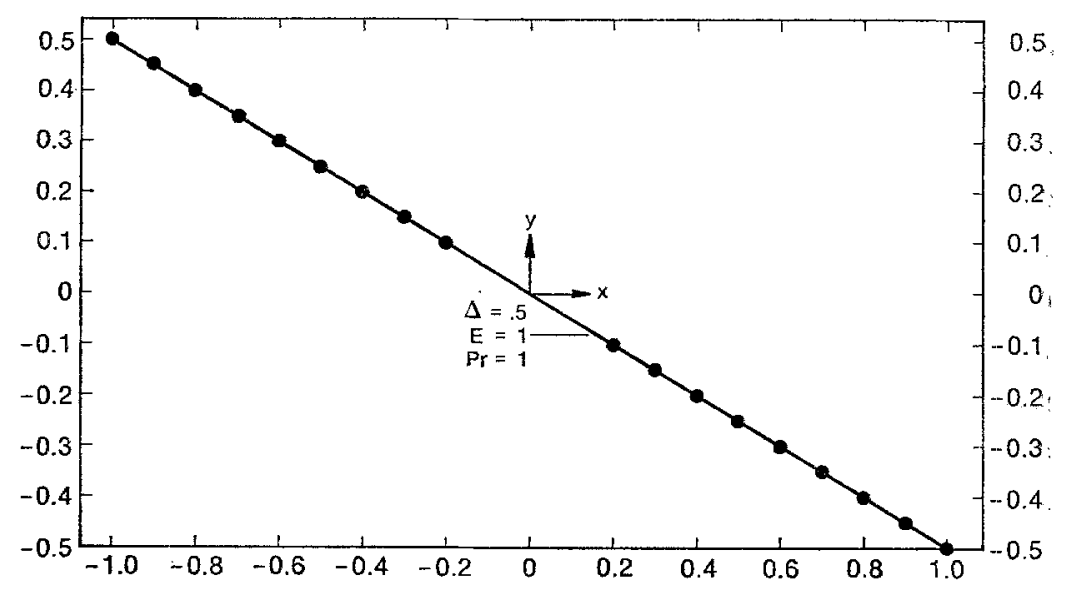

Fig. 2. Variation of temperature

Fig. 1 indicates the variation of the velocity profile $\bar{v}$ with $E, \operatorname{Pr}$, and $\delta$. Fig. 2 : depicts the temperature field $\bar{\theta}$. It is found that for the values of the parameters which have been considered, $\bar{\theta}$ varies linearly.

\section{References}

[1] Truesdell, C., Noll, W.: The non-linear field theories of mechanics (Handbuch der. Physik, III/3), Berlin-Heidelberg-New York: Springer 1965.

[2] Rivlin, R. S., Ericksen, J. I.: Stress deformation relations for isotropic materials. J. Ration. Mech. Anal. 4, 323-425 (1955). 
[3] Brace, R. W., Na, T. Y.: Natural convection flow of Powell-Eyring fluids between two vertical flat plates. ASME 67 WA/HT 25 (1967).

[4] Shenoy, A. V., Mashelkar, R. A.: Thermal convection in non-Newtonian fluids (Advances in heat transfer, Vol. 15). Academic Press 1982.

[5] Fosdick, R. L., Rajagopal, K. R.: Thermodynamics and stability of fluids of third grade. Proc. R. Soc. (London) A $3399,351-377$ (1980).

Prof. K. R. Rajagopal Department of Mechanical Engineering

University of Pittsburgh

Pittsburgh, PA 15261, U.S.A.
Dr.T. Y. Na

Department of Mechanical Engineering

University of Michigan

Deerborn Campus

Dearborn, MI 48128, U.S.A. 